\title{
Percussion test: description and diagnostic accuracy of a new manual test for bone marrow edema of the knee
}

\author{
Valerio Sansone ${ }^{1,2}$, Alessandro Galluzzo $^{3}$, Emanuele Maiorano ${ }^{2}$, Marina Benedetta Polatti ${ }^{4 *}$ and \\ Valerio Pascale ${ }^{1,2}$
}

\begin{abstract}
Background: Prompt diagnosis of bone marrow lesion (BML) is difficult but critical for correct treatment. Magnetic resonance imaging is the gold standard, although expensive and time consuming. Simple and reliable clinical test for BML detection is lacking. Aim of the study is to describe a new manual clinical test called Percussion Test (PT) and to statistically determine its diagnostic accuracy in BML, compared to MRI imaging.

Methods: After evaluation of the inclusion and exclusion criteria, 218 consecutive patients with unilateral knee pain and age comprised between 18 and 80 years old were enrolled in our observational prospective study. Informed consent was obtained for each patient. After medical history collection, PT was performed by a single operator as described. MRI was performed in the affected knee to detect the presence of BML. Coherence in PT and MRI assessment was evaluated in each quadrant of the knee via contingency tables, as sensitivity, specificity, NPV, PPV and diagnostic accuracy were calculated.
\end{abstract}

Results: No correlation with a positive PT was demonstrated for the covariables gender $(p=0.156)$, age $(p=0.272)$ and BMI $(p=0.639)$.

PT showed a sensitivity ranging from 60.6 (40.6-80.6) to 79.5 (63.0-96.0) and a specificity ranging from 85.7 (80.0-91.5) to 96.0 (93.1-98.9) depending on knee quadrant. Diagnostic accuracy ranged from 81.6 (75.9-86.6) to 89.4 (84.6-93.2), and $p$-value was $<0.00001$ in a chi-squared analysis for all quadrants.

Conclusions: PT showed sensitivity and specificity values that are comparable with other clinical tests routinely adopted in clinical practice. In the absence of other reliable clinical test, PT has the potential to become a useful bedside tool in the diagnosis and management of BMLS.

Keywords: Bone edema, Bone marrow lesion, Diagnostic accuracy, Knee pain, Physical examination, Knee, Prospective observational study, Sensitivity, specificity

\section{Background}

Over the last recent years, few topics have received as much attention from the orthopedic community as bone marrow edema (BME), at least judging by the remarkably

\footnotetext{
*Correspondence: marinapolatti@libero.it
}

${ }^{4}$ University of Milan, Milan, Italy

Full list of author information is available at the end of the article increasing amount of scientific publications on this issue.

Actually, BME is an interdisciplinary topic ranging from orthopedics to traumatology, radiology, rheumatology, physical medicine, up to internal medicine, endocrinology, diabetology, oncology, pediatrics, and beyond.

Compared to the first descriptions reported in the literature at the end of the ' 80 [1], a significant amount of data has been achieved, leading to a better original author(s) and the source, provide a link to the Creative Commons licence, and indicate if changes were made. The images or other third party material in this article are included in the article's Creative Commons licence, unless indicated otherwise in a credit line to the material. If material is not included in the article's Creative Commons licence and your intended use is not permitted by statutory regulation or exceeds the permitted use, you will need to obtain permission directly from the copyright holder. To view a copy of this licence, visit http://creativecommons.org/licenses/by/4.0/. The Creative Commons Public Domain Dedication waiver (http://creativeco mmons.org/publicdomain/zero/1.0/) applies to the data made available in this article, unless otherwise stated in a credit line to the data. 
comprehension of the mechanisms underlying the disease, although several aspects remain to be clarified. As evidence of this progress, at least three classifications [2-4] have been proposed and updated over the years. Moreover, the definition of the condition has also been modified, introducing the more appropriate term of Bone Marrow Lesion (BML) [5, 6].

However, while significant progress has been made in understanding pathophysiology and treatment, the same cannot be affirmed for the diagnosis, which continues to rely primarily on MRI (Magnetic Resonance Imaging). However, the limitations of MRI are well known: the cost of the examination, the relatively not ubiquitous distribution throughout the territory, and the length, sometimes very long, of the waiting lists. As a matter of fact, also because of the lack of symptom specificity, the correct diagnosis often is delayed and the condition may be misdiagnosed and treated incorrectly, if not untreated $[7,8]$.

Yet, prompt identification of BML would be crucial because its presence, beyond the disabling pain, is a well-known negative prognostic factor of progression of knee osteoarthritis with increased structural deterioration, cartilage damage, and reduced function [9-13].

Furthermore, some authors support the hypothesis that BML may represent an early stage of avascular necrosis $[14,15]$.

Thus, given the disabling symptoms of bone edema and its potential unfavorable evolution, a simple and reliable method to ascertain BMLs presence and to monitor their course would be highly desirable.

Therefore, we sought to develop a clinical test that would be easy to perform but, at the same time, would have sufficient reliability in diagnosing knee BMLs. Our test is based on the common clinical observation that bony areas affected by BMLs are painful on digital pressure. The explanation for this phenomenon could be reasonably due to the sudden rise of intramedullary pressure into the bone compartment due to the external percussive force. We named it "Percussion Test" (PT) and it is characterized by the manual percussion of the bony prominence of the knee to be evaluated. The test, in a way, takes inspiration from the classical semeiotics of the past, when instrumental diagnostics was not as developed as it is today, nor easily available. To date, no clinical test for diagnosing bone edema has been described, with the unique exception of the "tapping test" reported by Aigner et al. [16] However, performing this test requires the use of an apparatus consisting of a reflection hammer hinged to a support bracket, whose purpose would be to provide a reproducible percussive force. In brief, it is not exactly a simple test available in any clinical setting.
The aim of the present study was to evaluate the diagnostic accuracy of a manual test in detecting knee BMLs. The only tool needed is the examiner's bare hands.

\section{Materials and methods}

This was a single center observational prospective study in the context of a large academic hospital. In an ambulatory setting, initially, every patient underwent accurate anamnestic collection about past medical history and history of present illness. All patients referring knee issues was evaluated for study enrollment. Inclusion criteria were presence of unilateral knee pain and age comprised between 18 and 80 years. Exclusion criteria were presence of recent wounds around the knee and inflammatory processes involving overlying skin, including ecchymosis and hematoma. From October 2018 to October 2019, 218 patients were included in the study, 99 males and 119 females. Demographic data were collected. At the time of clinical assessment, the mean age was $55 \pm 13$,6years, and mean BMI was $25,9 \pm 4,8 \mathrm{~kg} / \mathrm{m}^{2}$. The clinical assessment was carried out by the same investigator during his usual clinical practice. Along with all other procedures, the investigator performed PT for detecting BML. PT was performed with the patient sitting and the calves hanging; the manual percussion was performed with two fingers and was targeted to the medial and lateral femoral condyle, and to the medial and lateral tibial plateau. The percussion of the femur was performed on the medial aspect of the medial condyle and on the lateral aspect of the lateral condyle, whereas the percussion of the tibia is performed on its anterior aspect, medially to the anterior tibial tuberosity for the medial plateau and just anterior to the fibular head for the lateral one. The test was always performed bilaterally, in order to define its positivity as the asymmetry of the pain perceived between the painful knee and the healthy one. In presence of a similar finding on both knees, the outcome was considered negative.

Subsequently, maximally 6 weeks after the clinical assessment, all patients underwent a knee MRI as the gold standard for detecting BML presence. Coronal, axial and sagittal T1-weighted sequences were performed, followed by coronal, axial and sagittal T2-weighted sequences and T2-weighted fat-suppressed sequences using a $1.5 \mathrm{~T}$ MRI scanner. The frontal planes of the T2-weighted images were divided into 4 quadrants, in order to define the exact location of BML [16]. Clinical evaluation data and MRI findings were collected in a database as presence or absence of BML. All subjects signed an informed consent form and the study was approved by a local ethics committee. The study was performed according to the Declaration of Helsinki principles for medical research involving human subjects. 
Data derived from the database were independently analyzed for each quadrant of the knee. Age and BMI were reported as mean standard deviation. The sensitivity, specificity, positive, and negative predictive values of the test were calculated for each quadrant, as well as diagnostic accuracy. CI95\% was reported. Chi-squared analysis of contingency tables was performed. A regression analysis was also performed to verify if the covariates age, gender, and BMI influenced the clinical test response. All statistical analyses were conducted with the IBM SPSS Statistics 24 software program (IBM Corporation, Armonk, NY), and the critical value for significance was set at $P<0.05$.

\section{Results}

218 patients were included in the study. 73 patients reported traumatic onset of pain (knee sprain, direct trauma), 38 patient reported acute (under 6 weeks) nontraumatic onset of pain while 107 patients reported chronic (over 6 weeks) non traumatic onset of pain. 79 patients showed a BML on MR imaging in 0 quadrants, 78 patients showed BML in 1 quadrant, 56 patients showed BML in 2 quadrants, 4 patients showed BML in 3 quadrants, 1 patient showed BML in all 4 quadrants of the knee. BML was located in the medial femoral condyle in 53 cases, the medial tibial plateau in 78 , the lateral femoral condyle in 33 , and the lateral tibial plateau in 42 cases. The observed overall BME prevalence in this

Table 1 Contingency tables for each knee quadrant. PT, percussion test; MRI, magnetic resonance

\begin{tabular}{llll}
\hline & & MRI positive & MRI negative \\
\hline Medial femoral condyle & PT positive & 34 & 21 \\
& PT negative & 19 & 144 \\
Lateral femoral condyle & PT positive & 20 & 10 \\
& PT negative & 13 & 175 \\
Medial tibial plateau & PT positive & 62 & 20 \\
& PT negative & 16 & 120 \\
Lateral tibial plateau & PT positive & 26 & 7 \\
& PT negative & 17 & 168 \\
\hline
\end{tabular}

population sample is $64.2 \%$, this observation being consistent with literature [17].

Contingency tables for each quadrant are reported in Table 1 . Sensitivity, specificity, positive, and negative predictive values are reported in Table 2. Chi-squared analysis of contingency tables is reported in Table 3.

No correlation with a positive PT was demonstrated for the covariables gender $(p=0.156)$, age $(p=0.272)$ and BMI $(p=0.639)$.

\section{Discussion}

PT showed high specificity values (85-96\%) and relatively low sensitivity values (60-79\%). Sensitivity and specificity values of PT shown in this study are comparable with the diagnostic accuracy of several functional evaluation tests widely adopted in everyday clinical practice. For example, the anterior drawer test for anterior cruciate ligament (ACL) insufficiency has a sensitivity ranging from $25 \%$ [18] to $91 \%$ [19] and a specificity ranging from $77 \%$ [20] to $100 \%$ [21] according to the literature. The Neer test, probably the most common routinely in use for the evaluation of shoulder impingement, has a sensitivity ranging from $33 \%$ [22] to $89 \%$ [23] and a specificity ranging from $30 \%$ [24] to $69 \%$ [25]. PT sensitivity shown in this study is lower than tapping test sensitivity reported by Aigner et al. [16] but positivity evaluation is conceptually different. They performed the tapping test with a hinged mallet, and the patient reported perceived pain on a visual analogical scale (VAS). VAS-reported pain is highly subjective, and it is very difficult to set a universal threshold to separate positive from negative results. Regarding this aspect, one of the advantages of

Table 3 Chi-squared analysis of contingency tables. $x^{2}$ is calculated with 1 degree of freedom and $N=218$. Significance is set at $p<.05$

\begin{tabular}{lll}
\hline & $\boldsymbol{x}^{2}$ & $\boldsymbol{p}$ \\
\hline Medial femoral condyle & 56.23 & $<0.00001$ \\
Lateral femoral condyle & 71.90 & $<0.00001$ \\
Medial tibial plateau & 90.75 & $<0.00001$ \\
Lateral tibial plateau & 85.67 & $<0.00001$ \\
\hline
\end{tabular}

Table 2 Sensitivity, specificity, positive, and negative predictive value, and accuracy values for each knee quadrant. The values are expressed in percentage (C195\% in brackets)

\begin{tabular}{llllll}
\hline & Sensitivity & Specificity & PPV & NPV & Accuracy \\
\hline Medial femoral condyle & $64.2(44.6-83.7)$ & $87.3(82.2-92.4)$ & $61.8(49.0-74.7)$ & $88.3(83.4-93.3)$ & $81.6(75.9-86.6)$ \\
Lateral femoral condyle & $60.6(40.6-80.6)$ & $94.6(91.3-97.9)$ & $66.7(49.8-83.5)$ & $93.1(89.8-96.7)$ & $89.4(84.6-93.2)$ \\
Medial tibial plateau & $79.5(63.0-96.0)$ & $85.7(80.0-91.5)$ & $75.6(66.3-84.9)$ & $88.2(82.8-93.7)$ & $83.5(77.8-88.2)$ \\
Lateral tibial plateau & $60.5(40.5-80.4)$ & $96.0(93.1-98.9)$ & $78.8(64.9-92.7)$ & $90.8(86.6-95.0)$ & 89.0 \\
& & & & & $(84.1-92.8)$ \\
\hline
\end{tabular}


our PT is the definition of a "positive" result. Patients are asked to compare the effect of the percussion stimulus received on both knees. PT is considered positive when the percussion is perceived differently in the examined knee, being either more painful or simply more pronounced. In this way, the patient performs an immediate internal analysis of the consequences of the mechanical stimulation, allowing the clinician to eliminate the bias deriving from the subjective threshold of pain.

The ideal application for the PT in clinical practice would be to implement it as a screening tool, to help identify a pool of patients with a heightened probability of BML, that are worthy or MRI imaging in terms of cost effectiveness. However, sensitivity is too low for the PT to be adopted as an effective screening tool.

Nevertheless, in the light of the high specificity we have observed, a positive result may be considered reliable. This implies that PT might be used in the early stages of the diagnostic pathway, to anticipate treatment, avoiding delays caused by the, often not immediate, MRI availability.

Moreover, a high negative predictive value of the PT may be employed to reduce the number of MRI controls during the follow-up period. The high negative predictive value (NPV) found (88-93\%) suggests that a patient diagnosed with BML but with a PT turned negative at followup, has a significant probability of being cured or, at least, to have experienced a significant reduction in BML's amplitude. Regarding the latter, previous studies have observed a significant correlation between the extent of BMLs and pain [26, 27].

In the absence of new anamnestic and clinical findings suggesting the presence of soft tissue lesions (e.g., meniscal tears), MRI might be avoided as the BML healing process can be monitored with a serial assessment of PT.

The major advantage of the PT lies in its simplicity and cost-effectiveness: it is intuitive, quick, and easy to perform. Moreover, no equipment is required and the learning curve seems to be really steep. Strength of the study is the large number of patient and the consistence of the data extracted, which comes from having all MRIs performed in one centre and the test performed by a single examiner.

A potential weakness lies in its reproducibility, although this is a common issue of every clinical manual test. In awareness of this, we intend to assess the inter and intra-observer variability in future studies.

\section{Conclusion}

In this retrospective observational study, the newborn PT showed moderate sensitivity (60-79\%) and good specificity $(87-96 \%)$. The most attractive aspects of the PT are its handiness and ease of execution since it can be replicated in every clinical context and no special tool is required. Overall, PT might have the right features to become a useful bedside tool in the diagnosis and management of BML.

\section{Abbreviations}

BME: Bone Marrow Edema; BML: Bone Marrow Lesion; MRI: Magnetic Resonance Imaging; PT: Percussion Test; ACL: Anterior Cruciate Ligament; VAS: Visual Analogical Scale; NPV: Negative Predictive Value; C195\%: Confidence Interval 95\%.

\section{Supplementary Information}

The online version contains supplementary material available at https://doi. org/10.1186/s12891-022-05028-y.

Additional file 1

\section{Acknowledgments}

Not applicable.

\section{Authors' contributions}

The idea backing this work is an original idea deriving from VS daily clinical activity. VS performed the procedure. AG, VP and EM contributed to research design and evaluated MRI obtained from each patient. AG, EM and MP performed analysis and interpretation of data. AG and EM drafted the first version of the paper. All authors contributed to the critical revision of the paper, and all authors read and approved the final and submitted manuscript.

\section{Funding}

No fundings were received for this study.

Availability of data and materials

All data generated or analysed during this study are included in the supplementary information files.

\section{Declarations}

\section{Ethics approval and consent to participate}

All procedures were in accordance with the ethical standards of the institutional and/or national research committee and with the 1964 Helsinki declaration and in its later amendments or comparable ethical standards. This study was approved by the local ethical committee (San Raffaele Hospital, Milan, Italy). Informed consent was obtained from all the individual participants included in the study.

\section{Consent for publication}

Not applicable.

\section{Competing interests}

The authors declare that they have no conflict of interest.

\section{Author details}

${ }^{1}$ Department of Orthopaedics, University of Milan, Milan, Italy. ${ }^{2}$ I.R.C.C.S. Istituto Ortopedico Galeazzi, Milan, Italy. ${ }^{3}$ Residency Program in Orthopaedics and Traumatology, University of Milan, Milan, Italy. ${ }^{4}$ University of Milan, Milan, Italy.

Received: 19 September 2021 Accepted: 4 January 2022

Published online: 18 January 2022

\section{References}

1. Wilson AJ, Murphy WA, Hardy DC, Totty WG. Transient osteoporosis: transient bone marrow edema? Radiology. 1988;167(3):757-60. 
2. Hofmann S, Kramer J, Breitenseher M, Pietsch M, Aigner N. Bone marrow edema in the knee. Differential diagnosis and therapeutic possibilities. Orthopade. 2006;35(4):463-77.

3. Starr AM, Wessely MA, Albastaki U, Pierre-Jerome C, Kettner NW. Bone marrow edema: Pathophysiology, differential diagnosis, and imaging. Acta Radiol. 2008;49(7):771-86.

4. Eriksen $\mathrm{EF}$, Ringe JD. Bone marrow lesions: a universal bone response to injury? Rheumatol Int. 2012;32(3):575-84.

5. Mayerhoefer ME, Breitenseher MJ, Kramer J, Aigner N, Norden C, Hofmann S. STIR vs. T1-weighted fat-suppressed gadolinium-enhanced MRI of bone marrow edema of the knee: computer-assisted quantitative comparison and influence of injected contrast media volume and aquisition parameters. J Magn Reson Imaging. 2005;22(6):788-93.

6. Hofmann S. The painful bone marrow edema syndrome of the hip joint. Wien Klin Wochenschr. 2005;117(4):111-20.

7. Arazi $M$, Yel M, Uguz B, Emlik D. Be aware of bone marrow edema syndrome in ankle arthroscopy: a case successfully treated with iloprost. Arthrosc J Arthrosc Relat Surg. 2006;22(8):909.e1-3.

8. Trepman E, King TV. Transient osteoporosis of the hip misdiagnosed as osteonecrosis on magnetic resonance imaging. Orthop Rev. 1992;21(9):1089-91, 1094-8.

9. Roemer FW, Guermazi A, Javaid MK, Lynch JA, Niu J, Zhang Y, et al. Change in MRI-detected subchondral bone marrow lesions is associated with cartilage loss: the most study. A longitudinal multicentre study of knee osteoarthritis. Ann Rheum Dis. 2009;68(9):1461-5.

10. Tanamas SK, Wluka AE, Pelletier JP, Pelletier JM, Abram F, Berry PA, et al. Bone marrow lesions in people with knee osteoarthritis predict progression of disease and joint replacement: a longitudinal study. Rheumatology. 2010;49(12):2413-9.

11. Felson DT, Mclaughlin S, Goggins J, Lavalley MP, Elon Gale M, Totterman S, et al. Bone marrow edema and its relation to progression of knee osteoarthritis background: while factors affecting the course of knee osteo. Ann Intern Med. 2003;139(5):330-7.

12. Raynauld JP, Martel-Pelletier J, Berthiaume MJ, Abram F, Choquette D, Haraoui B, et al. Correlation between bone lesion changes and cartilage volume loss in patients with osteoarthritis of the knee as assessed by quantitative magnetic resonance imaging over a 24-month period. Ann Rheum Dis. 2008;67(5):683-8.

13. Kothari A, Guermazi A, Chmiel JS, Dunlop D, Song J, Almagor O, et al. Within-subregion relationship between bone marrow lesions and subsequent cartilage loss in knee osteoarthritis. Arthritis Care Res. 2010;62(2):198-203.

14. Plenk H Jr, Hofmann S, Eschberger J, Gstettner M, Kramer J, Schneider W, Engel A. Histomorphology and bone morphometry of the bone marrow edema syndrome of the hip. Clin Orthop Relat Res. 1997;(334):73-84.

15. Berger CE, Kröner AH, Minai-Pour MB, Ogris E, Engel A. Biochemical markers of bone metabolism in bone marrow edema syndrome of the hip. Bone. 2003;33(3):346-51.

16. Aigner N, Meizer R, Meraner D, Becker S, Radda C, Landsiedl F. Tapping test in patients with painful bone marrow edema of the knee. Clin J Pain. 2008:24(2):131-4.

17. Guermazi A, Niu J, Hayashi D, Roemer FW, Englund M, Neogi T, Aliabadi P, McLennan CE, Felson DT. Prevalence of abnormalities in knees detected by MRI in adults without knee osteoarthritis: population based observational study (Framingham Osteoarthritis Study). BMJ. 2012;345:e5339.

18. Noyes FR, Bassett RW, Grood ES, Butler DL. Arthroscopy in acute traumatic hemarthrosis of the knee. Incidence of anterior cruciate tears and other injuries. J Bone Jt Surg - Ser A. 1980;62(5):687-95.

19. Braunstein E. Anterior cruciate ligament injuries: a comparison of arthrographic and physical diagnosis. Am J Roentgenol. 1982;138(3):423-5.

20. Warren RF, Marshall JL. Injuries of the anterior cruciate and medial collateral ligaments of the knee. A long-term follow-up of 86 cases--part II. Clin Orthop Relat Res. 1978;(136):198-211.

21. Torg JS, Conrad W, Kalen V. Clinical I diagnosis of anterior cruciate ligament instability in the athlete. Am J Sports Med. 1976;4(2):84-93.

22. Nakagawa S, Yoneda M, Hayashida K, Obata M, Fukushima S, Miyazaki Y. Forced shoulder abduction and elbow flexion test: a new simple clinical test to detect superior labral injury in the throwing shoulder. Arthrosc - J Arthrosc Relat Surg. 2005;21(11):1290-5.
23. Çaliş M, Akgün K, Birtane M, Karacan I, Çaliş H, Tüzün F. Diagnostic values of clinical diagnostic tests in subacromial impingement syndrome. Ann Rheum Dis. 2000;59(1):44-7.

24. Silva L, Andreu JL, Munoz P, Pastrana M, Millan I, Sanz J, et al. Accuracy of physical examination in subacromial impingement syndrome. Rheumatology. 2008;47(5):679-83.

25. Park HB, Yokota A, Gill HS, El Rassi G, EG MF. Diagnostic accuracy of clinical tests for the different degrees of subacromial impingement syndrome. J Bone Joint Surg Am. 2005;87(7):1446-55

26. Sansone V, Maiorano E, Pascale V, Romeo P. Bone marrow lesions of the knee: longitudinal correlation between lesion size changes and pain before and after conservative treatment by extracorporeal shockwave therapy. Eur J Phys Rehabil Med. 2019;55(2):225-30.

27. Driban JB, Price LL, Lo GH, Pang J, Hunter DJ, Miller E, et al. Evaluation of bone marrow lesion volume as a knee osteoarthritis biomarker - longitudinal relationships with pain and structural changes: data from the osteoarthritis initiative. Arthritis Res Ther. 2013;15(5):R112.

\section{Publisher's Note}

Springer Nature remains neutral with regard to jurisdictional claims in published maps and institutional affiliations.

Ready to submit your research? Choose BMC and benefit from

- fast, convenient online submission

- thorough peer review by experienced researchers in your field

- rapid publication on acceptance

- support for research data, including large and complex data types

- gold Open Access which fosters wider collaboration and increased citations

- maximum visibility for your research: over 100M website views per year

At BMC, research is always in progress.

Learn more biomedcentral.com/submissions 